International Journal of Advanced Chemistry, 2(1)(2014) $17-19$
International Journal of Advanced Chemistry
Journal home page: $\begin{gathered}\text { www.sciencepubco.com/index.php/IJAC } \\ \text { doi: } 10.14419 / \text { ijac.v2il.1625 } \\ \text { Research Paper }\end{gathered}$

\title{
A study of internal pressures of ternary and sub-binary liquid mixtures at various temperatures
}

\author{
N. Santhi*, G. Alamelumangai \\ Department of chemistry, government arts college, c.mutlur, Chidambaram, Tamilnadu, India \\ *Corresponding author E-mail: nsaanthi@gmail.com
}

\begin{abstract}
Internal pressures of the ternary and sub-binary mixtures of Benzene (1) + Hexane (2) $+n$-Butanol (3) have been computed from ultrasonic velocity measurements at different mole fractions in the temperature range of 300C-450C. The present work has been carried out to study the variation of internal pressure with temperature and composition of polar binary mixtures. The equation proposed by Andiappan et.al, for evaluating internal pressures of binary liquid mixtures has been modified and extended in the present work for the Ternary systems. The internal pressures computed with the help of the modified relation shows close agreement with observed values. The absolute deviation between the calculated and observed values from $1.15 \%$ to $1.9 \%$ for the ternary system studied.
\end{abstract}

Keywords: Ultrasonic velocity, density, molecular weight and internal pressure.

\section{Introduction}

The concept of internal pressure provides an excellent basis for examining solution phenomena. The first review of the subject by Richards1 appeared (in solution phenomena) in 1925, but the full potential of internal pressure as a structural probe did not become apparent until Hildebrand's2, 3 work a decade later. Extensive attempts have been made by a number of investigators4- 6 to calculate the internal pressure of liquids and liquid mixtures theoretically.

Internal pressure, being a measure of cohesive forces acting in a liquid, is sensitive to change of temperature, concentration and external pressure. Ultrasonic measurements provide a very important and accurate method for determination of internal pressure of liquids and the effects of the change in composition and temperature7-11. Internal pressure has been a subject of active interest during recent past and attempts have been made to show, the significance of internal pressure.

In an excellent article Dack12 reviewed the importance of solvent internal pressure and cohesion to solution phenomena. A review on the relationship between the intermolecular forces and properties was made by Kortum13; Barton14 discussed the relationship between internal pressure and molar volume in some depth.

The present work has been carried out to study the variation of internal pressure with temperature and composition of binary and ternary liquid mixtures. The equation proposed by S.C.Srivastava and N.Berkowitz15 was used to compute internal pressure from the measurement of ultrasonic velocity, density and molecular weight.

\section{Materials and methods:}

The liquids were purified as and when required as per the procedure recommended by Weissberger 16 .
Analar grade benzene supplied by B.D.H. India and guaranteed. Reagent grade $n$-Hexane of Japan product was redistilled after drying with sodium. The fraction boiling at $80.10 \mathrm{C}$ and $68.70 \mathrm{C}$ respectively were collected and stored. Guaranteed reagent grade n-Butanol was treated with calcium chloride and distilled. The fraction distilling at $97.10 \mathrm{C}$ was collected. The physical constants of pure liquids are given in table-1.

Table 1: The physical constants of pure liquids

\begin{tabular}{lllll}
\hline \multirow{3}{*}{ LIQUID } & $\begin{array}{l}\text { DENSITY } \\
\left(\mathrm{Kgm}^{-3}\right)\end{array}$ & \multicolumn{3}{l}{$\begin{array}{l}\text { BOILING POINT } \\
\left({ }^{\circ} \mathrm{C}\right)\end{array}$} \\
\hline & Expl. & Lit. & Expl. & Lit. \\
& $30^{\circ} \mathrm{C}$ & $25^{\circ} \mathrm{C}$ & $30^{\circ} \mathrm{C}$ & $25^{\circ} \mathrm{C}$ \\
n-HEXANE & 0.6493 & 0.6548 & 68.7 & 68.7 \\
BENZENE & 0.8675 & 0.8737 & 80.1 & 80.1 \\
n-BUTANOL & 0.8022 & 0.8020 & 117.8 & 117.7 \\
\hline
\end{tabular}

Ultrasonic velocities of pure liquids and liquid mixtures in the temperature range of $300 \mathrm{C}$ to $450 \mathrm{C}$ were measured using an ultrasonic interferometer operating at $3 \mathrm{MHz}$.

The density was determined at the experimental temperature using $10 \mathrm{ml}$ capacity specific gravity bottle immersed in a thermostatic bath (accuracy +0.010C). The volume of the bottle at the experimental temperatures, viz. 300C-450C was ascertained using doubly distilled water. The densities of water at these temperatures were obtained from literature.

\section{Theory and calculation:}

The internal pressure for pure liquids and mixtures at different temperatures were calculated using the equation (1) and (2) $\pi_{\mathrm{i}}=\frac{\mathrm{UD}}{\mathrm{K} \sqrt{\mathrm{M}}}$

Where 
$\pi_{\mathrm{i}}, \mathrm{U}, \mathrm{D}$ and $\mathrm{M}$ represent internal pressure, ultrasonic velocity, density and molecular weight of the pure component respectively. $\mathrm{K}$ was distinctly structure dependent constants.

$\left(\pi_{\mathrm{i}}\right)_{12}=\frac{\mathrm{U}_{12 \mathrm{D}_{12}}}{\left(\mathrm{X}_{1} \mathrm{~K}_{1}+\mathrm{X}_{2} \mathrm{~K}_{2}\right) \sqrt{\mathrm{M}_{12}}}$

Where

$\left(\pi_{\mathrm{i}}\right)_{12}, \mathrm{U}_{12}, \mathrm{D}_{12}$ and $\mathrm{M}_{12}$ represent internal pressure, ultrasonic velocity, density and molecular weight of the mixture respectively and $\mathrm{x}_{1}, \mathrm{x}_{2}$ are the molefractions of component 1 and 2 .

Suryanarayana and Kuppusamy ${ }^{17}$ derived an expression to account for the variation of internal pressure with concentration of the electrolyte which is of the form, internal pressure of

$\pi=\pi_{0}+A m^{2}+\mathrm{Bm}$

Where

$\pi$ is the internal pressure of the solution, $\pi_{0}$ is the internal pressure of the solvent, $\mathrm{m}$ is molality and $\mathrm{A}, \mathrm{B}$ are constants which are temperature dependent because of in adequacies of expression (3). It is modified and given by Andiappan et.al. ${ }^{18}$ in the form,

$\log \pi=x_{1} \log \pi_{1}+x_{2} \log \pi_{2}-\beta x_{1} x_{2}$

Where $\mathrm{x}_{1}$ and $\pi_{1}$ are the mole fraction and internal pressure of the component 1 and $x_{2}$ and $\pi_{2}$ are those of the component 2. The equation (4) containing only one constant $\beta$ has been correlating the experimental data.

In the present work, the extension of equation (4) for ternary system is attempted and the equation is written in the modified form,

$\log \pi=\mathrm{x}_{1} \log \pi_{1}+\mathrm{x}_{2} \log \pi_{2}+\mathrm{x}_{3} \log \pi_{3}+\mathrm{x}_{1} \mathrm{x}_{2}\left(\beta_{12}\left(\mathrm{x}_{1}-\mathrm{x}_{2}\right)\right)+$ $\mathrm{x}_{2} \mathrm{x}_{3}\left(\beta_{23}\left(\mathrm{x}_{2}-\mathrm{x}_{3}\right)\right)$

$+\mathrm{x}_{3} \mathrm{x}_{1}\left(\beta_{31}\left(\mathrm{x}_{3}-\mathrm{x}_{1}\right)\right)-\mathrm{C}\left(\mathrm{x}_{1} \mathrm{x}_{2} \mathrm{x}_{3}\right)$

Where

$\beta_{12}=$ binary interaction constant for 1,2 component

$\beta_{23}=$ binary interaction constant for 2,3 component

$\beta_{31}=$ binary interaction constant for 3,1 component

The constants $\beta_{12}, \beta_{23}$ and $\beta_{31}$ are determined from equation (4) and the constant $\mathrm{C}$ from equation (5). Eqn (5) containing the above constants have been employed for correlating the experimental data.

\section{Results and discussion:}

Table $2 \& 3$ summarizes the experimental determination of internal pressure values for Ternary as well as sub-binary systems. This is correlated through equations (4) and (5) in the temperature range $300 \mathrm{C}$ to $450 \mathrm{C}$. The constants $\beta$ and $\mathrm{C}$ have been determined through least square method at all temperatures. In both case, the binary and ternary interaction constants are not varied more when the temperature increase.

\section{Internal pressure in all binary systems:}

The absolute deviation between the experimental data and correlated values for the binary system varies from $0.028 \%$ to $0.1 \%$. The graphical method established the quantitative relations between internal pressure and temperature \& internal pressure and concentration.

Figure 1-3 shows a linear variation of internal pressure with Concentration of Alcohol in all various binary systems. Table (2) and (3) is evident that the internal pressure increase in concentration of alcohols, because at higher alcohol concentration hydrogen

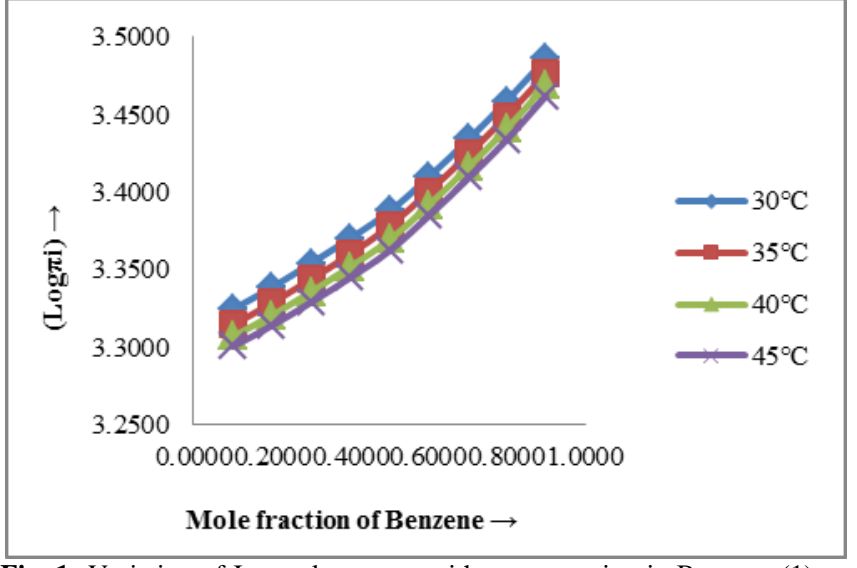

Fig. 1: Variation of Internal pressure with concentration in Benzene (1) + Hexane (2) at Various Temperature.

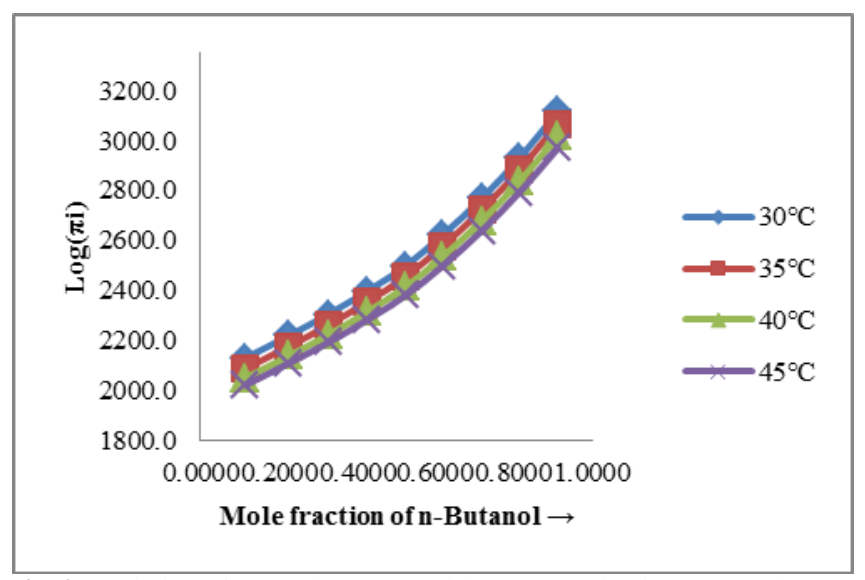

Fig. 2: Variation of Internal pressure with concentration in n-Butanol (1) + Hexane (2) at Various Temperature.

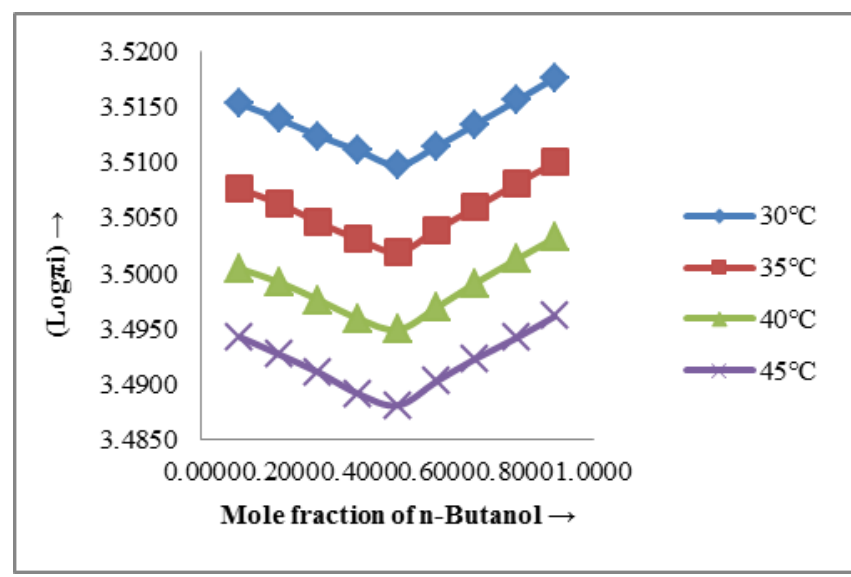

Fig. 3: Variation of Internal pressure with concentration in n-Butanol (1) + Benzene (2) at Various Temperature.

Bonding becomes predominant, resulting in the increase of the internal pressure. This suggests the close packing of the molecules inside, which may be brought about by increasing magnitude of interactions19, 20. Similar results are also evidenced by Rao et.al. 21 in o-Chlorophenol system.

The variation of Internal pressure with Temperature in all Various Binary systems is showed in Table (2) and (3) and also shows the falling of internal pressure with increase of temperature is the important observation in all the cases studied, because the cohesive forces between molecules becoming less with increase of temperature. Figure 4-6 shows a linear variation of internal pressure with the reciprocal of temperature for liquid mixtures in the temperature range $300 \mathrm{C}$ to $450 \mathrm{C}$. 


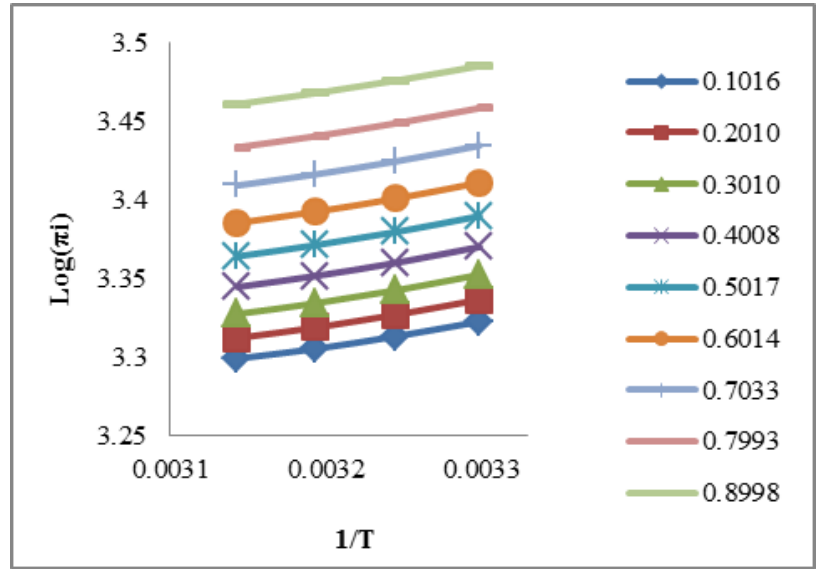

Fig. 4: variation of internal pressure with temperature in Benzene (1) + Hexane (2)

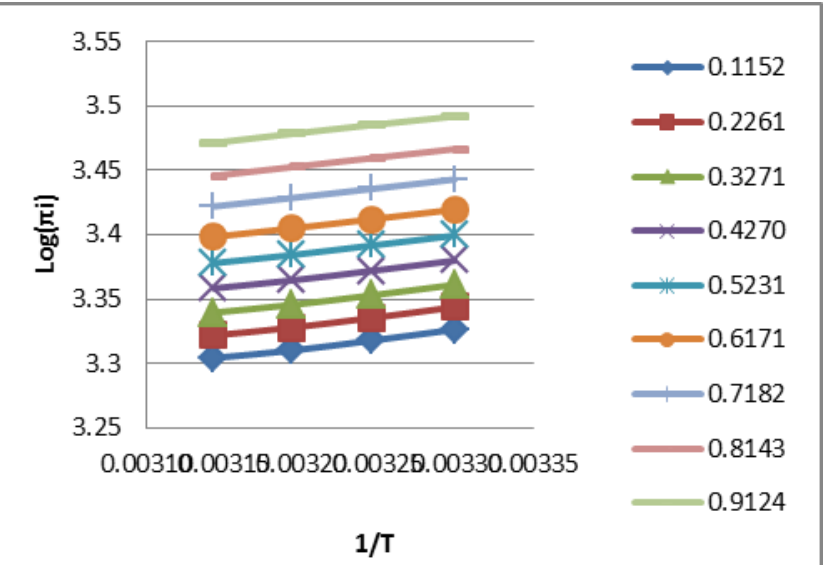

Fig. 5: variation of internal pressure with temperature in n-Butyl alcohol (1) + Hexane (2).

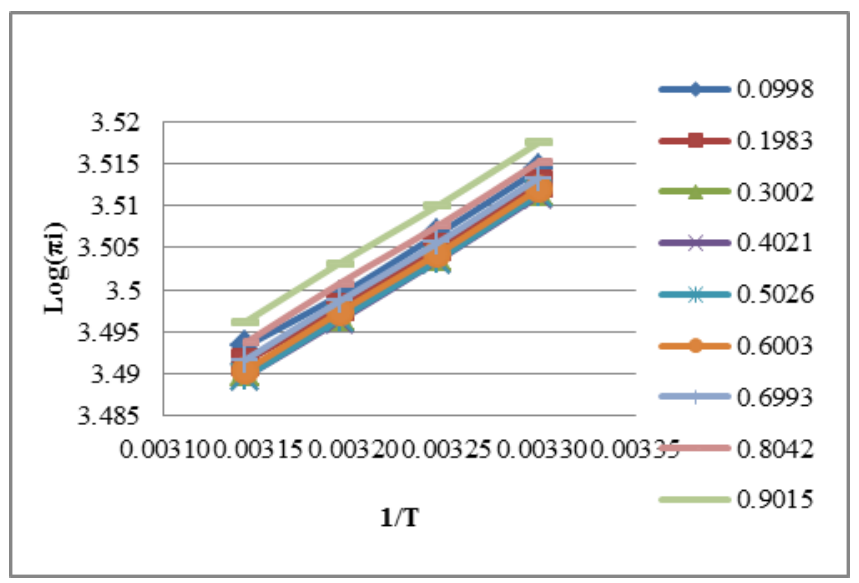

Fig. 6: variation of internal pressure with temperature in n-Butyl alcohol (1) + Benzene (2).

\section{Internal pressure in ternary system:}

The absolute average deviation between the experimental and correlated values for the Ternary system varies from $1.5 \%$ to $1.9 \%$. In the ternary systems the deviation is greater when the alcohol concentration is high because of the domination of hydrogen bonding at higher alcohol concentration, resulting in the increase of the deviation

\section{Conclusion}

From the present investigation, the absolute average deviation between the experimental data and correlated values for the Bi- nary system and Ternary system varies from $0.028 \%$ to $1.9 \%$, indicating the applicability of equation (4) and (5). A fairly good agreement has been found between experimental and theoretical values.

The effects of the concentration of Alcohol as a solute and the temperature effects on hydrogen bonds in the system are clearly seen. The role of hydrogen bonds and the structure of alcohols in interactions are observed. Finally we conclude that there exists a weak molecular association between the component molecules in Ternary mixtures and also in Sub-binary mixtures.

\section{References}

[1] T.W.Richards, Chem. Rev., 2, 315 (1925).

[2] J.H.Hildebrand and R.L.Scott, Solubility of Non-electrolytes, IIIrd Edn., Reinhold Publ. Corpn., Newyork, p.121, (1950).

[3] J.H.Hildebrand and R.L.Scott, Regular solutions, Prentice-Hall, Englewood Cliffs, New Jersy., (1962).

[4] C.V .Suryanarayana, J. Acout. Soc (India)., 4, 75 (1976).

[5] A.Ali \& M.Tariq, J.Chem.Res.(s), 4, 261(2006).

[6] J.D.Pandey, S.K.Shukla,J.Chhabra \& R.Dey, J. Indian Chem. Soc., 81, 962(2004).

[7] A.Dhana Lakshmi, J Acoust Soc India., 8(2), 29 (1980)

[8] J.D.Pandey \& R.L.Mishra, Acustica, 39, 1978 (2000).

[9] 9.P.S.Naidu, N.Prabhakara Rao \& K.Ravindra Prasad, Indian J. Pure \& Appl Ultrason., 39, 36(2002).

[10]Devadoss, Thairiyaraja \& Palaniappan, Indian J. Phys., 77B (6), 669 (2003).

[11]N.Prasad, Acustica., 74, 171 (1999).

[12]M.R.J.Dack, Chem. Soc. Rev., 4(2), 287, (1975).

[13]G.Kortum, Z.Electrochem., 42, 287, (1936).

[14] A.F.M.Barton, J.,Chem. Edu., 48(3), 156, (1971).

[15]S.C.Srivastava and N.Berkowitz, Canadian Journal of Chemistry, 41, 1787-1793 (1963).

[16]Weissberger, A Technique of organic chemistry Vol.7 (2nd edn), Interscience Newyork., (1955).

[17]C.V .Suryanarayana \& J.Kuppusamy, J. Acout. Soc (India)., 9(1) (1981).

[18] G.Amirthaganesan, S.Govinthasamy and An.Andiappan, Indian Journal of Chemistry, 25A 1023-1026 (1986).

[19] G.Arul \& L.Palaniappan, Indian J. Pure \& Appl. Phys., 39, 561 (2001).

[20]U.Srinivasulu \& P.R.Naidu, Indian J Pure \& Appl Ultrason., 17, 23 (1995).

[21]G.V.Rama Rao, A.Viswanathan Sarma, D.Ramachandran \& C.Rambabu, Indian J. Pure \& Appl. Phys., 43,602-608 (2005). 